\title{
Aço de alta dureza para aplicação balística: propriedades mecânicas
}

\author{
High hardness steel used as ballistic \\ armor: mechanical properties
}

\author{
Suzane de Sant'ana Oliveira ${ }^{1}$, Ricardo Pondé Weber ${ }^{1}$, \\ João Carlos Miguez Suarez ${ }^{1}$, Elson Renato Santos Souza ${ }^{2}$
}

\footnotetext{
${ }^{1}$ Seção de Engenharia Mecânica e Ciência dos Materiais - IME, CEP: 22290-270, Rio de Janeiro, Rio de Janeiro, Brasil.

${ }^{2}$ Departamento de Ciência e Tecnologia - EB, Rio de Janeiro, Rio de Janeiro, Brasil.

e-mail: susan.oliver@hotmail.com,rpweber@ime.eb.br,jmiguez@ime.eb.br, elsonqmb@yahoo.com.br
}

RESUMO

Blindagens balísticas são projetadas a resistir determinadas ameaças, que dependem da aplicação. Estas ameaças podem ser geradas por projéteis de armas de fogo ou estilhaços provenientes de explosões com diferentes energias cinéticas. Os materiais normalmente utilizados para este propósito são metálicos, não metálicos e compósitos, sendo seu desempenho avaliado por ensaios dinâmicos, tais como o realizado na Barra Hopkinson de Pressão e os balísticos no nível de proteção pretendido. Entretanto, o material pode ser previamente avaliado por ensaios mecânicos tradicionais, como por exemplo, ensaio de impacto e de dureza superficial, classificando-o como material passível de ser usado em proteção balística. Além disto, no processo de fabricação em série de viaturas militares de combate, o controle de qualidade dos materiais utilizados pode ser realizado através dos ensaios mecânicos citados, indicando se este atende os requisitos estabelecidos.

Devido sua relação custo/resistência balística, o aço ainda é o material mais utilizado na confecção de viaturas de combate. Algumas normas internacionais indicam as propriedades mecânicas mínimas, necessárias a uma determinada ameaça, que o material deve possuir para assegurar o seu desempenho balístico.

O objetivo deste trabalho foi o de avaliar a adequabilidade de um aço de alta dureza, produzido por siderúrgicas Brasileiras, proposto para a fabricação de viaturas militares com nível de proteção até a munição 7,62 $\mathrm{mm}$, através do ensaio de impacto, dureza e dobramento segundo o estabelecido na norma MIL-DTL-46100E. A análise da superfície de fratura, das amostras ensaiadas por impacto Charpy, foi realizada com o objetivo de correlacionar as propriedades mecânicas do material com o modo de fratura dos mesmos. Os resultados obtidos e as caracterizações da superfície de fratura sugerem que este aço não possui o comportamento mecânico adequado para o uso em viaturas de combate, porém sendo necessária a comprovação através do ensaio balístico.

Palavras-chave: Aço de alta dureza HHA; blindagem balística; resistência balística.

\section{ABSTRACT}

Ballistic armors are designed to resist specific threats, which depend on the application. These threats can be generated by firearm projectiles or fragments from explosions with different kinetic energies. The materials usually used for this purpose are metallic, nonmetallic and composite, being evaluated by dynamic tests, such as the Hopkinson Pressure Bar and the ballistic ones at the desired level of protection.

However, the material can be previously evaluated by traditional mechanical tests, such as impact and surface hardness tests, classifying it as a material that can be used in ballistic protection. Also, during of serial production of military combat vehicles, the quality control of the applied material can be done by mechanical tests like the mentioned above to verify compliance with the established requirements.

Due to its cost / ballistic resistance ratio, steel is still the most used material to produce of combat vehicles. There are international standards that show the minimum mechanical properties that a steel need to get specific ballistic protection.

The objective of this work was to evaluate the compliance of a high hardness steel, produced by Brazilian 
steel company, proposed for the production of military vehicles with protection level up to ammunition 7.62 $\mathrm{mm}$, through the impact, hardness and bending tests with the MIL-DTL-46100E. The analysis of the fracture surface of the samples tested by Charpy impact was carried out to correlate the mechanical properties of the material with the fracture mode. The results obtained and the characterization of the fracture surface suggest that the studied steel does not have the mechanical behavior suitable for the use in combat vehicles, been necessary to prove it through the ballistic test.

Keywords: High hardness steel; ballistic armor; ballistic resistance.

\section{INTRODUÇÃO}

Materiais metálicos, não metálicos e compósitos podem ser utilizados para produção de blindagens balísticas, dependendo do nível de proteção pretendido e mobilidade necessária à sua aplicação [1,2].

Como a relação peso/resistência balística é de grande importância em blindagens móveis, estas são categorizadas segundo os níveis de energia cinética a que terão que resistir. No caso de proteção a projéteis, as normas de avaliação do desempenho balístico definem uma ou duas munições representativas de uma classe de projéteis de energia cinética equivalente [3]. Assim, dependendo da norma utilizada, os níveis de proteção balístico podem ser divididos em 4 ou mais grupos.

Embora os materiais metálicos possuam a maior densidade dentre os mencionados, estes ainda são os mais utilizados em veículos militares devido principalmente a sua boa relação custo/benefício [4, 5]. Blindagens metálicas projetadas para resistir a munições perfurantes, como por exemplo, $7.62 \mathrm{~mm}, 0.30 \mathrm{~mm}$ e 0.45 ACP $(11,5 \mathrm{~mm})$ devem possuir a capacidade de desestabilizar/fragmentar a ponta do projetil, diminuindo o poder de perfuração, absorver sua energia cinética, bem como evitar o estilhaçamento da blindagem, isto é, devem apresentar alta dureza e boa tenacidade, propriedades antagônicas para um aço. Estas propriedades podem ser alcançadas através de uma composição química adequada e tratamento térmico compatível. Atualmente, o aço de alta dureza denominado HHA (High Hardness Armor) é o mais utilizado para este propósito quando tratado termicamente por têmpera e revenimento.

A resistência balística de um material deve ser avaliada segundo normas específicas que garantam o nível de proteção requerido, porém suas propriedades mecânicas, tais como tenacidade e dureza, que indicam a adequabilidade do material ao uso preterido, podem ser previamente testadas através de ensaios mecânicos $[6,7,8]$.

No caso do aço de alta dureza estudado, para o nível de proteção até a munição 7,62 mm, os requisitos mecânicos estão estabelecidos na norma MIL-DTL-46100E, que prevê: uma dureza Brinnell entre 477-534 HB ( 48 e 54 HRC) na superfície de impacto balístico, uma absorção de energia no ensaio de impacto Charpy a $-40^{\circ} \mathrm{C}$, por unidade de área, de no mínimo 20 e $23 \mathrm{~J} / \mathrm{m}^{2}$ nas direções transversal e longitudinal e da não observância de trincas superficiais durante o ensaio de dobramento em ângulo de $90^{\circ}$ do corpo de prova $[9$, $10,11]$.

Este trabalho teve como objetivo comparar os resultados obtidos nos ensaios de impacto, dureza e dobramento do aço estudado, produzido por siderúrgicas Brasileiras, com os requisitos estabelecidos segundo a norma MIL-DTL-4600E, além de analisar o modo de fratura dos corpos de prova de impacto através da microscopia eletrônica de varredura (MEV).

\section{MATERIAIS E MÉTODOS}

O material estudado foi o aço de alta dureza para emprego em proteção balística, submetido ao processo de refusão por escória eletrocondutora, de acordo com a norma MIL-A-46173 (1994). Este material foi fornecido sob a forma de chapas laminadas, nas dimensões de $3000 \mathrm{~mm} \times 3000 \mathrm{~mm}$ x $8 \mathrm{~mm}$, tratadas termicamente por meio de têmpera a partir de $1000^{\circ} \mathrm{C}$ e revenimento a $400^{\circ} \mathrm{C}$, com o objetivo de obter uma microestrutura de martensita revenida (Figura 1). A composição química é apresentada na Tabela 1 [12].

Para a realização dos ensaios mecânicos, a chapa foi cortada com o auxílio de um jato de água, a fim de não alterar a microestrutura do material.

Tabela 1: Composição do aço HHA (\% em massa) [12]. 


\begin{tabular}{c|c|c|c|c|c|c|c|c}
\hline $\mathbf{C}$ & $\mathbf{M n}$ & $\mathbf{S i}$ & $\mathbf{N i}$ & $\mathbf{C r}$ & $\mathbf{M o}$ & $\mathbf{C u}$ & $\mathbf{T i}$ & $\mathbf{P + S}$ \\
\hline 0,31 & 0,42 & 0,93 & 0,57 & 0,76 & 0,30 & 0,05 & 0,03 & 0,009 \\
\hline
\end{tabular}

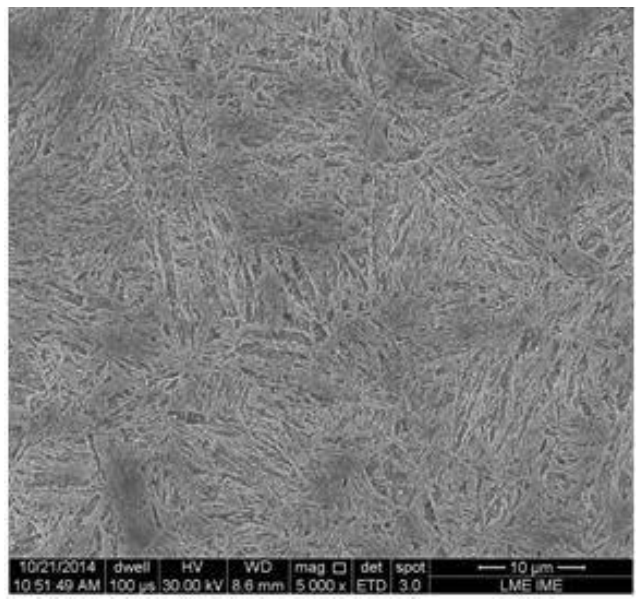

Figura 1: Microfotografia da microestrututra de martensita revenida em 5000x.

\subsection{Ensaio de impacto Charpy}

$\mathrm{O}$ ensaio de impacto Charpy foi realizado a $-40^{\circ} \mathrm{C}$, em corpos de provas com dimensões não padronizadas devido à espessura da chapa, em uma máquina de ensaios de impacto marca Wolpert, modelo PW 30, com capacidade máxima de $300 \mathrm{~J}$, de acordo com a norma ASTM E-23 [13]. A obtenção da temperatura de ensaio foi conseguida através de uma mistura de nitrogênio líquido e álcool. $10 \mathrm{~mm})$.

A Figura 2 apresenta um desenho esquemático das dimensões dos corpos de prova $(55 \mathrm{~mm} \mathrm{X} 8 \mathrm{~mm} \mathrm{X}$

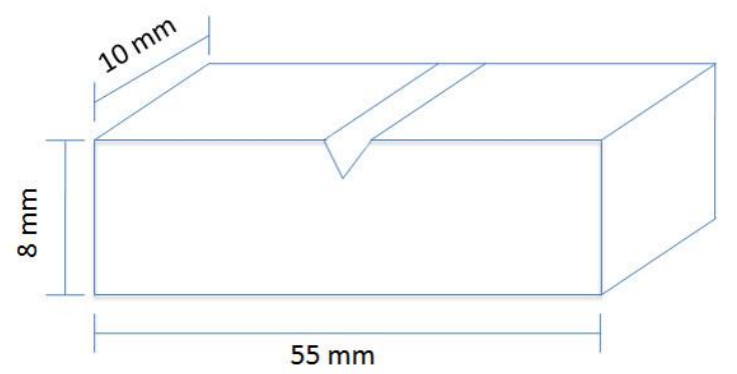

Figura 2: Desenho esquemático dos corpos de prova de impacto Charpy.

Foram testados ao todo, 10 corpos de provas, sendo cinco retirados na direção de laminação (longitudinal) e cinco transversalmente a mesma, todos com entalhe em V na direção da espessura da chapa, conforme a Figura 3.

Determinou-se para cada corpo de prova uma energia de impacto por unidade de área e foi calculado o valor médio para cada direção na chapa. Os resultados foram comparados aos requisitos apresentados na norma MIL-DTL-46100E [10].




Figura 3: Corpos de prova nas direções: (a) longitudinal e (b) transversal.

\subsection{Ensaio de dureza Rockwell}

$\mathrm{O}$ ensaio de dureza Rockwell foi realizado à temperatura ambiente, utilizando a escala $\mathrm{C}$ (penetrador de diamante e uma carga de $150 \mathrm{kgf}$ ) de acordo com a norma ASTM E-18 [14], nas duas superfícies da chapa bem como na sua espessura, a $2 \mathrm{~mm}$ de profundidade e a $3 \mathrm{~mm}$ profundidade.

Foram realizadas 7 indentações em linha em cada condição acima especificada conforme o desenho esquemático apresentado na Figura 4.

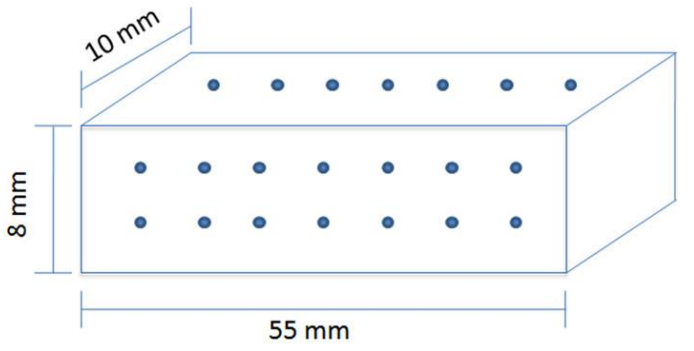

Figura 4: Identificação das regiões onde foram realizadas as indentações de dureza Rockwell C.

\subsection{Ensaio de Dobramento}

O ensaio de dobramento foi realizado a temperatura ambiente em 6 corpos de prova, de dimensões $300 \mathrm{~mm} x$ $10 \mathrm{~mm}$ x $8 \mathrm{~mm}$, usinados na direção transversal a direção de laminação, conforme apresentado na Figura 5 [15], de acordo com a norma ASTM E290-1 [16].

O corpo de prova, apoiado em 2 (dois) roletes espaçados de $80 \mathrm{~mm}$, foi dobrado pela ação de um cutelo com $60 \mathrm{~mm}$ de espessura e raio de curvatura de $30 \mathrm{~mm}$ até um ângulo de $90^{\circ}$. A velocidade de ensaio foi de $15 \mathrm{~mm} / \mathrm{s}$. O ensaio teve como objetivo verificar a ocorrência de trincas no material, após o dobramento [15].

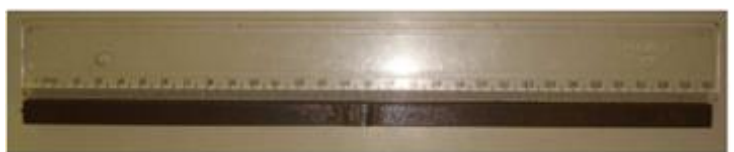

Figura 5: Fotografia do corpo de prova de dobramento [15].

\subsection{Análise da superfície de fratura por MEV}

A análise da superfície de fratura dos corpos de prova de impacto foi realizada por meio de microscopia eletrônica de varredura (MEV). Este exame procurou determinar os mecanismos de fratura predominantes no aço HHA produzido pelo impacto dinâmico do martelo, relacionando com os valores de energia absorvida pelo material no ensaio de impacto Charpy.

\section{RESULTADOS E DISCUSSÃO}




\subsection{Ensaio de impacto Charpy}

A Tabela 2 apresenta os valores de energia absorvida por unidade de área do ensaio de impacto Charpy, nas direções longitudinal e transversal.

Tabela 2: Valores das energias absorvidas (Joules), determinados no ensaio de impacto Charpy do aço HHA, nas direções transversal e longitudinal.

\begin{tabular}{|c|c|c|c|c|c|}
\hline \multicolumn{6}{|c|}{ DIREÇÃO DE EXTRAÇÃO DOS CORPOS DE PROVA (CP's) } \\
\hline \multicolumn{3}{|c|}{ TRANSVERSAL } & \multicolumn{3}{|c|}{ LONGITUDINAL } \\
\hline $\mathrm{CP}$ & Energia $(J)$ & $\mathrm{J} / \mathrm{cm}^{2}$ & $\mathrm{CP}$ & Energia (J) & $\mathrm{J} / \mathrm{cm}^{2}$ \\
\hline $\mathrm{CP} 1$ & $-*$ & $-*$ & $\mathrm{CP} 1$ & 20 & 29 \\
\hline $\mathrm{CP} 2$ & 21 & 24 & $\mathrm{CP} 2$ & 20 & 27 \\
\hline $\mathrm{CP} 3$ & 20 & 23 & $\mathrm{CP} 3$ & 20 & 26 \\
\hline $\mathrm{CP} 4$ & 29 & 25 & $\mathrm{CP} 4$ & 20 & 26 \\
\hline CP5 & 20 & 28 & CP5 & 19 & 27 \\
\hline Total: & 90 & 100 & Total: & 99 & 135 \\
\hline Média: & $22,5 \pm 2$ & 25 & Média: & $19,8 \pm 1$ & 27 \\
\hline
\end{tabular}

Observa-se que a energia absorvida por unidade de área dos corpos de prova individualmente, bem como a média, tanto na direção longitudinal quanto na direção transversal, são superiores ao valor mínimo requerido pela norma [10], indicando que o material estudado apresenta a tenacidade necessária ao desempenho balístico pretendido, ou seja, o material possui a capacidade de absorver a energia cinética do projétil, não permitindo que ocorra a penetração completa no material.

\subsection{Ensaio de dureza}

A Tabela 3 mostra os valores de dureza Rockwell C medidos nas duas superfícies da chapa (Face 1 e Face 2) e na espessura.

Tabela 3: Representação gráfica dos valores de dureza Rockwell C do aço estudado.

\begin{tabular}{c|c|c|c|c|c|c|c|c|c}
\hline \multirow{2}{*}{$\begin{array}{c}\text { LOCAL DE } \\
\text { INDENTAÇÃO }\end{array}$} & \multicolumn{10}{c}{ DUREZA ROCKWELL C } \\
\cline { 2 - 11 } & $\mathbf{1}$ & $\mathbf{2}$ & $\mathbf{3}$ & $\mathbf{4}$ & $\mathbf{5}$ & $\mathbf{6}$ & $\mathbf{7}$ & Média & S (desvio) \\
\hline FACE 1 & 35 & 37 & 39 & 33 & 38 & 36 & 31 & 35,6 & $\pm 3,41$ \\
\hline FACE 2 & 25 & 29 & 31 & 32 & 29 & 32 & 26 & 29,1 & $\pm 2,82$ \\
\hline 2 mm de profundidade & 46 & 50 & 47 & 49 & 49 & 48 & 54 & 49,0 & $\pm 2,6$ \\
\hline 3 mm de profundidade & 47 & 45 & 48 & 49 & 49 & 49 & 49 & 48,0 & $\pm 1,53$ \\
\hline & & & & & & \multicolumn{2}{c}{ Média total: } & 40,4 & \\
\hline
\end{tabular}

Os resultados mostram que as superfícies da chapa apresentaram valores abaixo do mínimo recomendado pela norma, porém a 2 e $3 \mathrm{~mm}$ de profundidade os valores observados estão acima do recomendado, indicando que a microestrutura produzida pelo tratamento térmico propicia a dureza necessária ao bom desempenho balístico do material. Este decréscimo apresentado na superfície do material nos levou a supor uma possível descarbonetação do material durante o tratamento térmico realizado, sendo esta suposição confirmada através do ensaio metalográfico realizado na espessura da chapa atacada com Nital $2 \%$, como apresentado na Figura 6 [17]. 


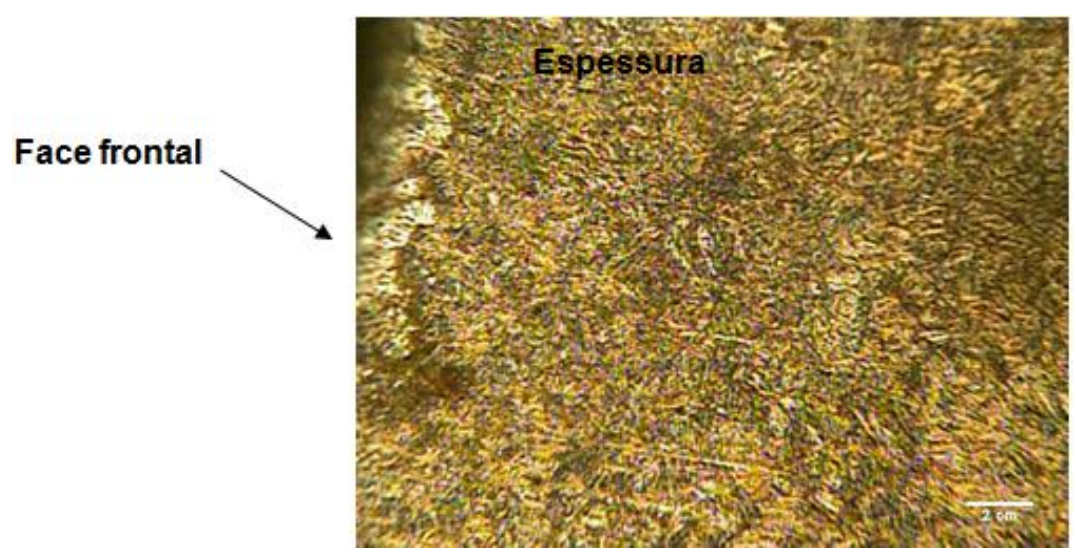

Figura 6: Micrografia ótica da espessura atacada com Nital 2\% (675x), evidenciando a descarbonetação na face frontal.

Estes valores de dureza da superfície, abaixo do recomendado, indicam que o material não possui o requisito mínimo para quebrar a ponta do projetil e consequentemente diminuir o poder de perfuração do mesmo $[6,18,19]$.

\subsection{Ensaio de dobramento}

A Figura 7 apresenta o aspecto dos corpos de prova, após o ensaio de dobramento.

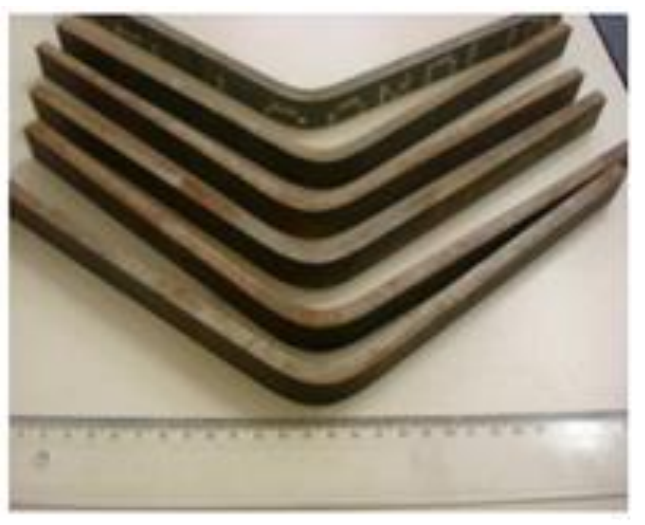

Figura 7: Corpos de prova do aço HHA, como recebidos, ensaiados por dobramento a $90^{\circ}$ [15].

Os corpos de prova apresentaram uma recuperação elástica significativa durante o ensaio, o que produziu um ângulo de deformação final, inferior a $90^{\circ}$.

As amostras não fraturaram e não apresentaram trincas. Este comportamento está em conformidade com a norma MIL-DTL-46100E.

\subsection{Análise fratográfica por MEV}

As Figuras 8 e 9 apresentam as macrografias do ensaio de impacto Charpy realizadas a $-40^{\circ} \mathrm{C}$ nas direções transversal e longitudinal, respectivamente.

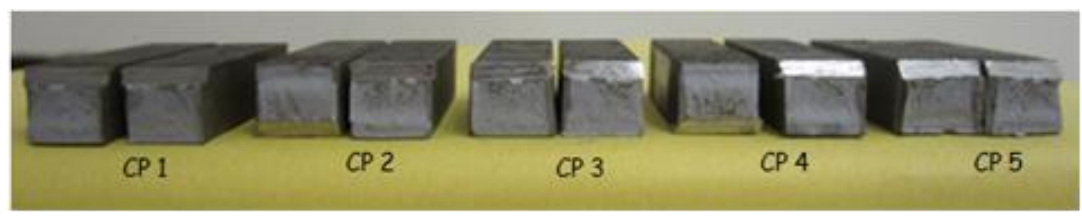

Figura 8: Aspectos macroscópicos das superfícies de fratura de corpos de prova ensaiados por impacto a $-40^{\circ} \mathrm{C}$ na direção transversal. 


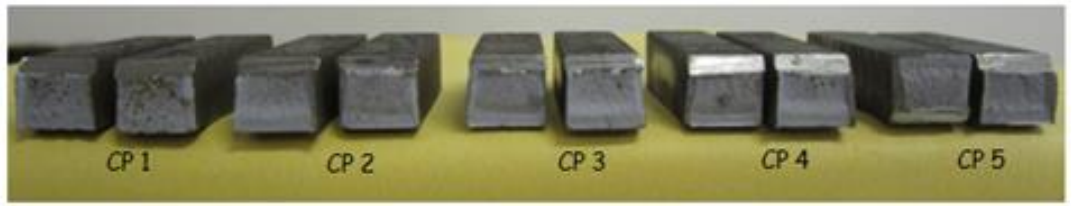

Figura 9: Aspectos macroscópicos das superfícies de fratura de corpos de prova ensaiados por impacto a $-40 \mathrm{C}$ na direção longitudinal.

A figura 10 apresenta as regiões características de fratura do corpo de prova de impacto.

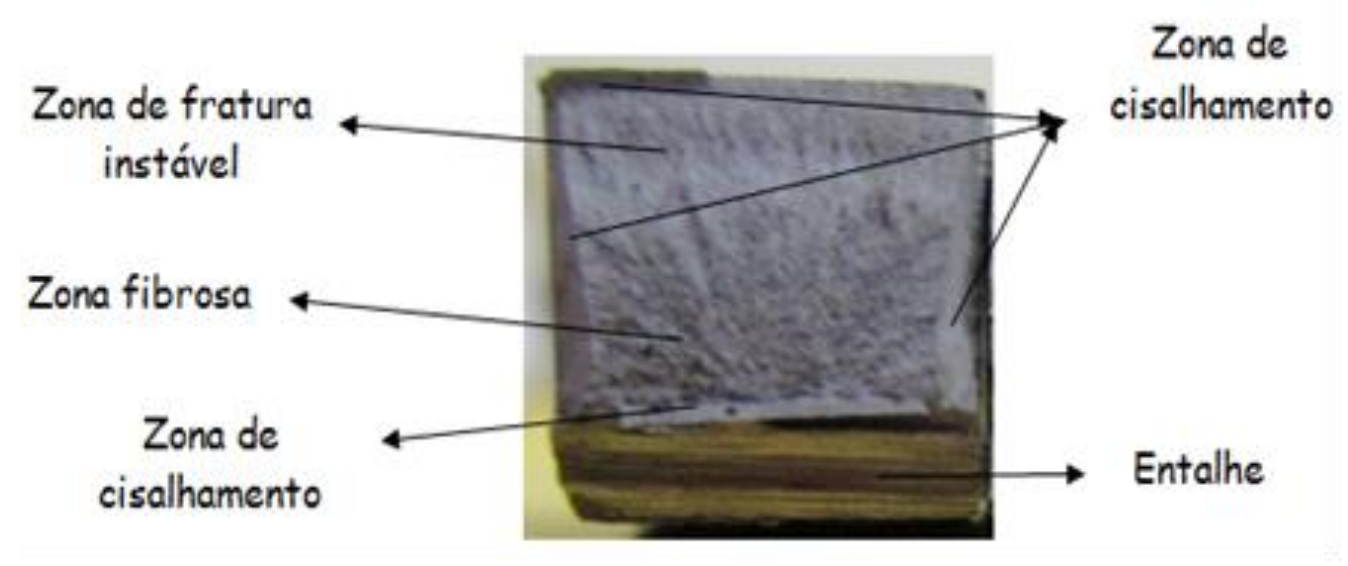

Figura 10: Visualização das zonas na fratura do CP de impacto.

Observa-se próximo ao entalhe uma zona de cisalhamento, seguida da zona fibrosa, que percorre grande parte da amostra, terminando em uma zona de fratura instável. As bordas do corpo de prova apresentaram características da zona de cisalhamento, devido à baixa temperatura a qual o ensaio foi realizado [21, 22].

A Figura 11 apresenta com maiores detalhes devido à ampliação, a zona de fratura instável (Fig.11(a)) e a zona de cisalhamento (Fig.11(b)) onde se pode perceber uma mistura dos aspectos de fratura dúctil e frágil do material, com predominância de mecanismos dúcteis [23].

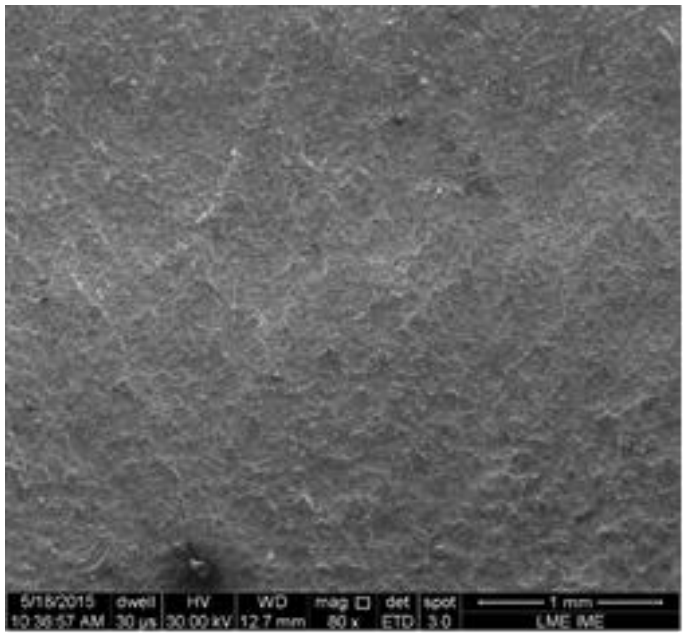

(a)



(b)

Figura 11: Micrografias do CP3 (longitudinal) em (a) 80x e (b) 200x. 


\section{CONCLUSÕES}

Os resultados apresentados demonstram que o material, através do impacto Charpy, possui tenacidade suficiente para absorver a energia cinética proveniente do projetil 7,62 $\mathrm{mm}$ e conseqüentemente não permitir a penetração completa do alvo. Este resultado é confirmado pela análise da superfície de fratura do corpo de prova de impacto, que demonstra um comportamento mais dúctil do que frágil do material ao ser submetido a um carregamento dinâmico, por apresentar mais mecanismos de deformação plástica do que de clivagem, embora o material tenha sido submetido ao tratamento térmico de têmpera e revenido proposto para obtenção da microestrutura martensítica revenida.

No ensaio de dobramento foi observada uma significativa recuperação elástica, demonstrando ser o material resiliente, o que contribui para a dissipação da energia cinética além de não apresentar trincas após o ensaio.

Porém o ensaio de dureza demonstrou que apesar do material ter alcançado a dureza recomendada pela norma, ou seja, possuir dureza suficiente para romper a ponta do projetil e assim diminuir seu poder de penetração, a superfície, devido à descarbonetação ocorrida durante o tratamento térmico, apresentou dureza inferior a recomendada, sugerindo que este material não seja apropriado para o nível de proteção balística pretendido.

Entretanto o tratamento térmico sob atmosfera inerte pode ser a solução para esta não conformidade.

\section{AGRADECIMENTOS}

Os autores agradecem ao Exército Brasileiro (EB) e as agências de financiamento nacional (CNPq, CAPES and FAPERJ) por patrocinar essa pesquisa.

\section{BIBLIOGRAFIA}

[1] LAKSHMI, L., NANDA KUMAR, C.G., "Investigations on the performance of metallic and composite body armors", Procedia Technology, v.25, pp.145-163, 2016. DOI: https://doi.org/10.1016/j.protcy.2016.08.094.

[2] FANG, H., PALTA, E., GUTOWSKI, M., "Numerical simulation of high-speed impacts involving metallic and non-metallic materials", International Journal of Computational Methods and Experimental Measurements, v.6, n.3, pp. 463-475, 2018. DOI: 10.2495/CMEM-V6-N3-463-475.

[3] ARMOR, "Personal Body", Ballistic Resistance of Personal Body Armor, NIJ Standard-0101.04. 2008.

[4] HAZELL, P.J.,"'The development of Armour Materials", Military Technology - MILTECH, v.4, pp. 5462, 2006. http://www.dtic.mil/dtic/aulimp/citations/gsa/2006_153341/131362.html

[5] GORNI, "Aços avançados de alta resistência: microestrutura e propriedades mecânicas", Corte e conformação de metal, Dez. 2008. http://www.gorni.eng.br/Gorni_CCM_Dez2008.pdf

[6] MEYERS, M., A., Dynamic behavior of materials. John Wiley \& Sons, 1994.

[7] JENA, P.K., SIVA KUMAR, K., KRISHNA, V.R., et al., "Studies on the role of microstructure on performance of a high strength armour steel', Engineering Failure Analysis, v. 15, n.8, pp. 1088-1096, 2008. DOI: https://doi.org/10.1016/j.engfailanal.2007.11.011

[8] BOAKYE-YIADOM, S., KHAN, A.K., BASSIM, N., "A Systematic Study of grain refinement during impact of 4340 steel”, Materials Science \& Engineering A, v. 605, p. 270-285, 2014. DOI:

https://doi.org/10.1016/j.msea.2014.03.066.

[9] SPECIFICATION, Detail. Armor plate, steel, wrought, homogeneous (for use in combatvehicles and for ammunition testing). US Army Research Laboratory, pp. 1-46, 2009.

[10] SPECIFICATION, Detail. Armor Plate, Steel, Wrought, High-Hardness. US Army Research Laboratory, pp. 1-30, 2008.

[11] SPECIFICATION, Detail, Armor steel, plate, wrought, (ESR), US Army Research Laboratory, 1994.

[12] STHIFFER TECNOLOGIA LTDA e FUNDAÇÃO RICARDO FRANCO (FRF), Relatório Técnico do Contrato $\mathrm{N}^{\circ}$ TP 24/2008, Resultados finais de ensaios de aços de composições experimentais, Rio de Janeiro, RJ, 2008.

[13] AMERICAN SOCIETY FOR TESTING AND MATERIALS. ASTM Standard Test Methods for Notched Bar Impact Testing of Metallic Materials: E 23-12c. 
[14] AMERICAN SOCIETY FOR TESTING AND MATERIALS. ASTM Standard Test Methods for Tension Testing of Metallic Materials: E8/E8M - 13a.

[15] SOUZA, E. R. S., Estudo do comportamento microestrutural e balístico de um aço de blindagem após soldagem, Dissertação de M. Sc, IME, Rio de Janeiro, RJ, Brasil, 2014.

[16] AMERICAN SOCIETY FOR TESTING AND MATERIALS. ASTM Standard Test Methods for Bend Testing of Material for Ductility: E290 - 2013.

[17] AMERICAN SOCIETY FOR METALS. Metals Handbook, Fractography, v. 12, 10 ed., 1993.

[18] BACKMAN, M.E, GOLDSMITH, W., "The mechanics of penetration of projectiles into targets", International Journal Engng. Sci., v. 16, pp. 1-99, 1987. DOI: https://doi.org/10.1016/0020-7225 (78)90002-2.

[19] SHAHKARAMI, A., CEPUS, E., VAZIRI, R., et al., "Material responses

to ballistic impact. Lightweight ballistic composites - Military and low enforcement applications". A. Bhatnagar (Ed.). Chapter 3, p.72-100, Boca Raton: CRC Press, 2006.

[20] GARCIA, A., "Ensaios dos Materiais" / Amauri Garcia, Jaime Alvares Spim, Carlos Alexandre dos Santos, 2 ed., Rio de Janeiro, LTC, 2012.

[21] WEBER, R. P., CHAWLA, K. K., SUAREZ, J.C. M. "Influence of notch orientation and temperature on the impact behavior of a dual hardness steel composite", Materials Science and Engineering: A, v. 580, pp. 279-287, 2013. DOI: https://doi.org/10.1016/j.msea.2013.05.026.

[22] KIM, H., PARK, J., KANG, M., LEE, S., "Interpretation of Charpy Impact energy characteristics by microstructural evolution of dynamically compressed specimens in three tempered martensitic steels", Materials Science \& Engineering A, v. 649, pp.57-67, 2016. DOI: https://doi.org/10.1016/j.msea.2015.09.099

[23] SILVA, P.S.C. "Microfotografia, Introdução às características topográficas correspondentes aos diversos micromecanismos de fratura", Metalurgia, v.27. n.166, pp.641-652, 1971.

\section{ORCID}

Ricardo Pondé Weber

Suzane de Sant' Ana Oliveira

Elson Renato Santos Souza

João Carlos Miguez Suarez https://orcid.org/0000-0002-7431-8316

https://orcid.org/0000-0003-4139-4870

https://orcid.org/0000-0003-3263-0296

Não informado 